\title{
Mehr Regulierung in der Medizin - Qualitätsschub oder Innovationshemmnis?
}

\section{Ruth Amstein, \\ Alexander Breitenstein, \\ Thomas F. Lüscher}

Zurich Heart House -

Foundation for Cardiovascular

Research, Zurich und

Klinik für Kardiologie,

HerzKreislaufZentrum,

UniversitätsSpital Zürich

Korrespondenz:

Dr. Ruth Amstein

Zurich Heart House -

Foundation for

Cardiovascular Research

Moussonstrasse 4

CH-8091 Zürich

ruth.amstein[at]usz.ch

\section{Ist zu viel Ethik unethisch?}

Professor Thomas Lüscher begrüsste die zahlreichen Teilnehmer mit der Feststellung, dass die klinische Forschung wie auch die molekulare Grundlagenforschung für den Wirtschaftsstandort Schweiz von grosser Bedeutung sei - nicht nur stellten sie kluge Köpfe für die Industrie und das Gesundheitswesen zur Verfügung, sie schafften auch Wissen, neue Therapien, Patente und damit neue Arbeitsplätze und Produkte. Die Forschung findet heute zunehmend in einem internationalen Umfeld mit wachsender Konkurrenz statt, deshalb ist nicht nur ihre Qualität, sondern zunehmend auch der Zeitfaktor bedeutsam.

Es braucht eine Regulierung der Forschung, damit Studienobjekte (unabhängig, ob Mensch oder Tier) nicht zu Schaden kommen. Für die Regulierung gilt aber grundsätzlich, was Paracelsus für Medikamente vor Jahrhunderten festhielt: "Alles ist Gift, nur die Dosis macht's, ob etwas Gift ist oder nicht.» Mit anderen Worten, kann ein Zuviel an Ethik auch unethisch sein, und ein Zuviel an Regulierung kann nicht nur sichern, sondern auch behindern. In der Schweiz hat sich eine enorme Regulierungswut entwickelt, die zu den strengsten Vorschriften weltweit geführt hat. Insbesondere im Bereich Tierversuche und Stammzellforschung, aber auch bei der ethischen und administrativen Prüfung von klinischen Projekten ist heute ein beträchtlicher Zeitaufwand erforderlich. Neben den relativ hohen Kosten für klinische Studien in der Schweiz, den eher langsamen Rekrutierungszeiten und international vergleichsweise geringen Patientenzahlen kann der Forschungsplatz Schweiz unter diesen Bedingungen trotz hoher Qualität der Prozesse und des Personals langfristig nicht an der Spitze mithalten.

\section{Ein vernünftiges Verhältnis zwischen staat- licher Regulierung und Eigenverantwortung ist anzustreben}

FDP-Nationalrätin Gabi Huber stellte fest, dass in der Schweiz zunehmend eine Entwicklung von einem liberalen Staat zu einem Leistungs- und Wohlfahrtsstaat stattfindet. Da der Staat somit immer mehr Aufgaben übernimmt, nimmt zwangsläufig auch die Regulierung zu. Auch gesellschaftliche Entwicklungen bewirken eine Zunahme der staatlichen Regulierung. Zu Herzen gehende Tagesaktualitäten führen regelmässig zu einer Forderung nach gesetzgeberi-

\section{Plus de régulation en médecine - en faveur de la qualité ou au détriment de l’innovation?}

La neuvième «Cardiovascular Roundtable» (CARTA), qui s'est tenue le 24 octobre 2012 à l'Hôpital universitaire de Zurich, avait pour objectif de réunir les responsables de l'industrie technique médicale et pharmaceutique, le corps médical, les assureurs-maladie et les politiciens dans le but d'instaurer un dialogue ouvert entre les différents partenaires de la santé. Animée par le Prof. Peter Suter, président du Swiss Medical Board, et par le Prof. Thomas F. Lüscher, la table ronde s'est intéressée à la régulation en médecine et à ses effets sur l'innovation.

La régulation en général mais en particulier en médecine doit rester modérée, sans quoi elle ne ferait que bloquer l'innovation, au détriment des patients et de la place scientifique suisse.

schen Schritten auf Bundesebene. Die daraus resultierende Gesetzesflut hat negative Folgen auf die Qualität der Gesetzgebung und führt zur grotesken Entwicklung, dass Gesetze immer öfter bereits revidiert werden müssen, bevor sie überhaupt in Kraft getreten sind. Für die Rechtsanwendung wird der Normendschungel fast undurchdringlich.

Huber plädierte auf mehr Eigenverantwortung des Individuums und fasste ihr Referat wie folgt zusammen. «Nur mit geteilter Verantwortung zwischen Staat und Bürgern, dem nötigen Augenmass und genügend Zeit kann sinnvolles Recht entstehen. Der Bereich Medizin und Forschung bildet dabei keine Ausnahme, jedoch stellt sich infolge des ständigen Fortschritts die Regulierungsfrage gerade hier permanent. Weil damit oft auch ethische Fragen ver- 
bunden sind, ist die Abwägung zwischen detaillierter Regulierung oder eben einem Regulierungsverzicht in diesem Bereich besonders herausfordernd und zwingend.»

\section{Bringt uns das Swiss Medical Board (SMB) weiter?}

Professor Urs Metzger, Mitglied des Expertenrats des SMB, zeigte die Bedeutung dieses Fachgremiums in der schweizerischen Gesundheitspolitik. Es befasst sich mit dem Mehrwert einer Behandlungsform unter Betrachtung der Kosten-Wirksamkeits-Relation, denn der medizinische Fortschritt ist in der Regel mit Kostensteigerungen verbunden. Das SMB erarbeitet Entscheidungsgrundlagen für den optimalen Einsatz von medizinischen Leistungen unter Berücksichtigung einer hohen Qualität bei effizientem Ressourceneinsatz. Metzger strich hervor, dass das SMB keine Zulassungsbehörde sei. Die methodischen Ansätze für die Leistungsbeurteilung enthalten eine medizinische, ökonomische, ethische und rechtliche Komponente. Die Behandlungen sollen entsprechend der Vorgabe durch das KVG Art. 32 wirksam, zweckmässig und wirtschaftlich sein.

\section{Health Technology Assessment (HTA): Chance für eine bessere Entscheidungsfindung?}

Daniel Herren, Chefarzt Handchirurgie an der Schulthess Klinik, zeigte den HTA-Prozess am praktischen Beispiel des Morbus Dupuytren auf. Während sich der Patient früher einer aufwendigen Operation unterziehen musste, ist heute als Alternative die Behandlung mit einer Spritze mit Kollagenase möglich. In der Entscheidungsfindung - Operation versus Infiltration - müssen verschiedene medizinische und ökonomische Aspekte gegeneinander abgewogen und die verschiedenen Anspruchsgruppen befriedigt werden. Der HTA-Prozess kann in komplexen Situationen mehr Klarheit für Behandlungsrichtlinien bringen. Es findet ein Assessment unter Erfassung des individuellen Mehrnutzens, der Evidenz, dem sozialen Nutzen und einer ökonomischen Bewertung statt. Auf dieser Grundlage wird ein «Appraisal» mit Grenzen, Regeln und Empfehlungen erarbeitet und am Schluss eine Entscheidung zuhanden der Zulassungsbehörde gefällt. Herren ist überzeugt, dass der HTA-Prozess eine grosse Chance darstellt, wovon sämtliche Stakeholder profitieren können.

\section{Ethik und Innovation - ein Widerspruch?}

Gemäss Ruth Baumann-Hölzle, Institutsleiterin Dia$\log$ Ethik, ist Ethik die Wissenschaft der Moral. Moralisches Denken entsteht dann, wenn die Selbstverständlichkeit des Handelns zerbricht, wobei Moral und damit die Vorstellung dessen, was als Handlung richtig sein soll, von individuellen wie auch gesellschaftlichen Wertvorstellungen beeinflusst wird. Immanuel Kant (1724-1804), der das ethische Denken der Moderne entscheidend prägte, verwendete als Erster den Begriff der Autonomie. Aufgrund seiner Willensfreiheit kann der Mensch alles, was ist, fortan hinterfragen. «Das, was ist, muss nicht sein.»

Welche Rolle hat die Ethik bei den heutigen Entwicklungen im Gesundheitssystem? Das, was ist, ist im Hinblick auf allgemeinverbindliche Werte wie Menschenwürde und Gerechtigkeit zu hinterfragen. So müssen aus ethischer Sicht die WZW-Kriterien des KVGs, Wirksamkeit, Zweckmässigkeit und Wirt-

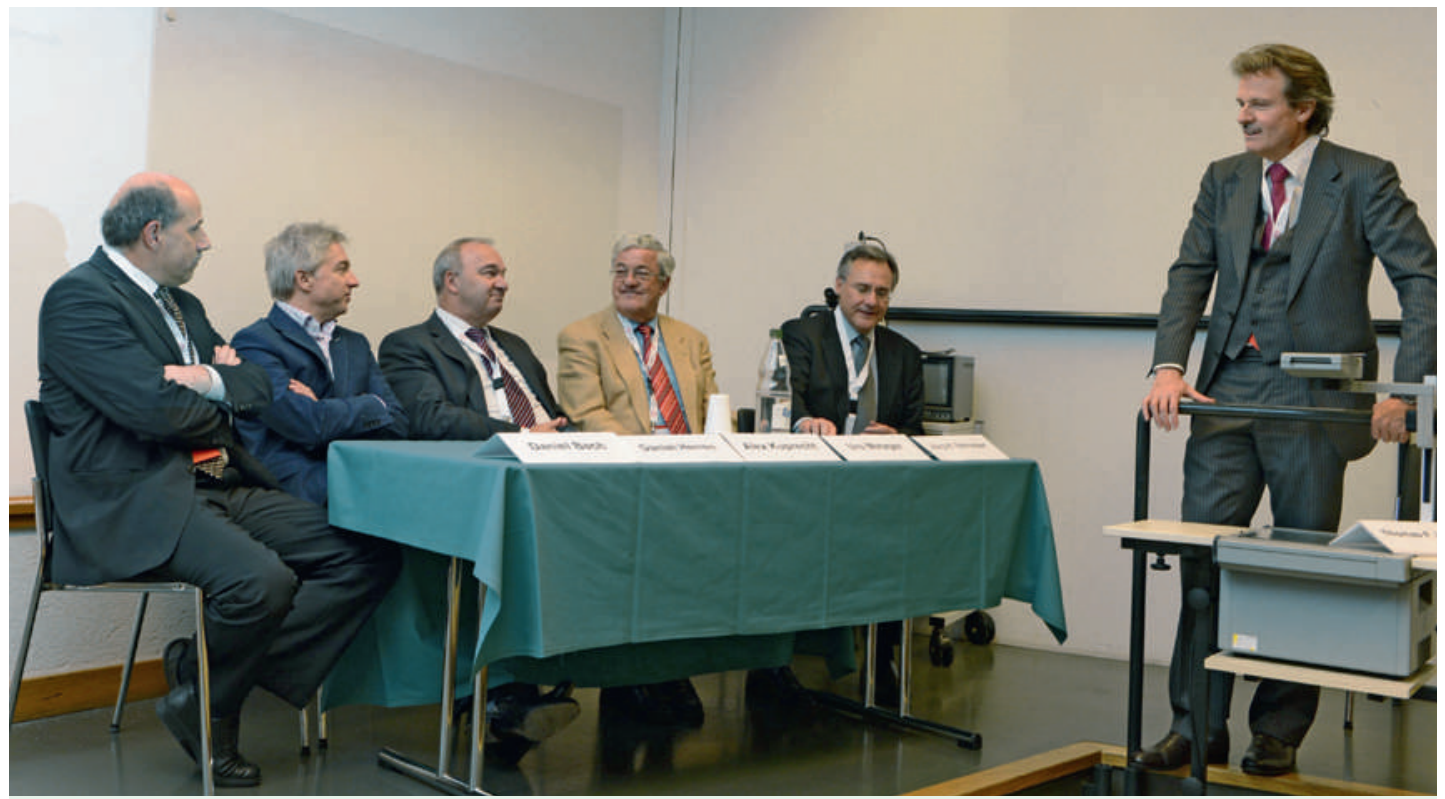

Engagierte Diskussion der Gesundheitspartner an der Podiumsdiskussion. (Foto: Sam Rogers, Zurich Heart House) 
schaftlichkeit normativ gefüllt werden, damit sie ethisch vertretbar operationalisiert werden können. Ebenso ist der Begriff «Innovation», der aus den Wirtschaftswissenschaften stammt, ethisch zu klären. Neuerungen sind nicht per se Innovationen. Im Gesundheitswesen besteht derzeit die Gefahr, dass eine Neuerung nur aufgrund von ihrem ökonomischen Nutzenpotential als Innovation beurteilt wird, wobei der leidende Mitmensch aus dem Blickfeld verloren zu gehen droht. Das Gesundheitswesen hat laut Baumann-Hölzle einen hohen Innovationsbedarf in struktureller und vor allem politischer Hinsicht.

\section{Die Swissmedic als Gesundheitspolizei}

Der Direktor von Swissmedic, Jürg H. Schnetzer, beschrieb seine Behörde als Gesundheitspolizei, die dafür sorgt, dass nur qualitativ einwandfreie, sichere und wirksame Heilmittel auf dem Markt sind. Die Behörde ist im Sicherheits- und Wirtschaftsaufsichtsbereich tätig. Es stellt sich dabei immer die Frage, wie viel Regulierung ein Zustand der relativen Sicherheit im Zusammenhang mit Heilmitteln braucht und wie hoch die Toleranzschwelle in der Forschung liegt.

\section{Die Überregulation ist ein deutlicher Nachteil}

\section{der Schweiz gegenüber dem Ausland.}

Bezüglich des gesellschaftlichen Einflusses auf die Regulierung sagte Schnetzer: «Wir leben in einer Post-Trust-Society. Vieles wird in Frage gestellt, obwohl kein Anlass dafür besteht.» Kleinere Vorkommnisse führen zu Grundsatzdiskussionen und Unsicherheiten, die mit noch mehr Regulierung behoben werden wollten. Die Folge davon seien immer mehr Vorstösse im Parlament, die Formulierungen enthielten wie «In den Medien war zu erfahren ... wie stellt sich der Bundesrat dazu?» Es dürfe nicht die Rolle der Medien, insbesondere auch der Social Media sein, durch Beschleunigung und Dramatisierung von Ereignissen zu mehr Regulierung beizutragen und in anderem Zusammenhang die zunehmende Regulierung zu kritisieren.

\section{Code of Conduct Ärzte/Industrie: Wie viel Regulierung ist sinnvoll?}

«Non-Compliance - der Vorhof zur Hölle!» Unter diesem Motto begann die Break-out-Session mit einem Input-Referat von Lorenz Borer (Novartis Schweiz) unter Mitwirkung von Melchior Buchs (FASMED) und Dr. Dieter Grauer (Scienceindustries). Der «Code of Conduct» zwischen Ärzten und Industrie wird in einer Art von Imperialismus von den USA an Westeuropa und die Schweiz diktiert. Die Pharmaindustrie scheint im Fokus von Untersuchungen wegen Korruption und Bestechung zu stehen, deren Ursprung in Risikoländern, wo die Korruption auf der Tagesordnung steht, zu suchen ist. Als Folge der Globalisierung wird nicht nur in den Risikoländern, sondern aus Angst global überreguliert. Die Leidtragenden dieser Überregulierung sind die Ärzte selbst. Die Finanzierung von Fortbildungsbeiträgen durch uneingeschränkte Grants durch die Industrie könnte in Frage gestellt werden. Medizinalkongresse, die ein Wirtschaftszweig in Städten und Tourismusregionen sind, sind in ihrer Existenz gefährdet. Schweizer Orte werden nicht zuletzt wegen des hohen Frankens zu Luxusdestinationen deklariert, die durch europäische Pharma- und Medizinaltechnikverbände für Kongresse nicht mehr bewilligt werden. Um Vorurteilen zu begegnen, muss man bei Vergütungen an Ärzte volle Transparenz schaffen und die Massnahmen kommunikativ begleiten.

\section{Wie viel Regulierung braucht die Forschung?}

Die zweite Break-out-Session wurde von Prof. Simon Hoerstrup (Unispital Zürich), PD Christian Matter (Unispital Zürich) und Jürg H. Schnetzer geleitet. Die Teilnehmer diskutierten über den Prozess der Forschungsprojektentwicklung, von dessen Planung bis zur erfolgreichen Publikation in einer Fachzeitschrift. Hervorgehoben wurde dabei der internationale Konkurrenzdruck und entsprechend die Bedeutung einer gerechten und länderübergreifend vergleichbaren Regulation, die dazu dienen soll, dass die Objektivität und Transparenz über ein Produkt beziehungsweise über ein Resultat gewährleistet ist, denn Ängste und Unsicherheit in der Bevölkerung führen zur Forderung nach mehr Regulierung.

\section{Selbstbewusster Umgang mit den Ressourcen}

In der abschliessenden Podiumsdiskussion, an der neben den Referenten auch SVP-Ständerat Alex Kupprecht, Daniel Bach (Medtronic Schweiz) sowie Prof. Peter Suter teilnahmen, wurden die einzelnen Positionen noch einmal festgehalten. Urs Metzger hielt fest, dass jeder Fehler nach Kontrolle rufe, denn wir wollen in einer «No-risk»-Gesellschaft leben. Bach sieht in der Überregulation einen deutlichen Nachteil der Schweiz gegenüber dem Ausland, denn die Bewilligungen für internationale Studien dauern oft zu lange. Schnetzer schlug vor, mit Augenmass zu regulieren, wobei die Prozesse Risiko-basiert ablaufen sollten. Wir müssen lernen, mit Restrisiken umzugehen. Das Gremium war sich einig, dass die Schweiz selbstbewusst mit ihren Ressourcen Bildung, Forschung und Qualität der Hochschulen umgehen sollte. Der Universität und der ETH Zürich gelingt es immer noch, hervorragende Forscher aus der ganzen Welt anzuziehen. Bach stellte fest, dass die Schweiz für Firmen nach wie vor ein attraktiver Standort mit gutausgebildeten Leuten, Steuervorteilen und einem stabilen politischen Umfeld darstelle. 